\title{
Legendre transform structure and extremal properties of the relative Fisher information
}

\author{
R. C. Venkatesan ${ }^{a, *}$ A. Plastino ${ }^{b}$ \\ a Systems Research Corporation, Aundh, Pune 411007, India \\ ${ }^{\mathrm{b}}$ IFLP, National University La Plata \& National Research Council (CONICET) \\ C. C., 727 1900, La Plata, Argentina
}

\begin{abstract}
Variational extremization of the relative Fisher information (RFI, hereafter) is performed. Reciprocity relations, akin to those of thermodynamics are derived, employing the extremal results of the RFI expressed in terms of probability amplitudes. A time independent Schrödinger-like equation (Schrödinger-like link) for the RFI is derived. The concomitant Legendre transform structure (LTS, hereafter) is developed by utilizing a generalized RFI-Euler theorem, which shows that the entire mathematical structure of thermodynamics translates into the RFI framework, both for equilibrium and non-equilibrium cases. The qualitatively distinct nature of the present results vis-á-vis those of prior studies utilizing the Shannon entropy and/or the Fisher information measure (FIM, hereafter) is discussed. A principled relationship between the RFI and the FIM frameworks is derived. The utility of this relationship is demonstrated by an example wherein the energy eigenvalues of the Schrödinger-like link for the RFI is inferred solely using the quantum mechanical virial theorem and the LTS of the RFI.
\end{abstract}

Key words: Relative Fisher information, generalized RFI-Euler theorem, Legendre transform structure, Schrödinger-like link, inference, energy eigenvalues.

PACS: 05.20.Gg; 89.70.Cf; 02.30.Sa

\footnotetext{
* Corresponding author.

Email addresses: ravi@systemsresearchcorp.com (R. C. Venkatesan), plastino@fisica.unlp.edu.ar (A. Plastino).
} 


\section{Introduction}

The Fisher information measure (FIM, hereafter) [1-3]

$$
I[f]=\int_{\Re^{n}} f(\mathbf{x})\left(\frac{\partial \ln f(\mathbf{x})}{\partial \mathbf{x}}\right)^{2} d \mathbf{x}=\int_{\Re^{n}} \frac{1}{f(\mathbf{x})}\left(\frac{\partial f(\mathbf{x})}{\partial \mathbf{x}}\right)^{2} d \mathbf{x}
$$

where $\mathbf{x}$ is a vector $\left(\frac{\partial f\left(x_{i}\right)}{\partial x_{j}}=0 ; i \neq j\right)$, has played a prominent role in statistics, information theory, physics, and allied disciplines. In addition to the applications cited in Ref. [3], the FIM has also been successfully employed in areas as diverse as biology, social science, econophysics, and encryption of covert information amongst a number of other applications (eg. see [4]). The relative Fisher information (RFI, hereafter) defined by $[5,6]$

$$
\Im[f \mid g]=\int_{\Re^{n}} f(\mathbf{x})\left|\nabla \ln \frac{f(\mathbf{x})}{g(\mathbf{x})}\right|^{2} d \mathbf{x}
$$

where $|\bullet|^{2}$ is the square norm, has been primarily studied within the context of mathematical physics and optimal transportation in statistical physics (eg. Refs. $[7,8]$ and the references therein). Note that $\Im[f \mid g]=0$ when $f(\mathbf{x})=g(\mathbf{x})$, and $\Im[f \mid g] \neq \Im[g \mid f]$ (asymmetric). An alternate form of the $n$-dimensional RFI has been suggested by Carlen and Soffer [9] for a Gaussian $g(x)$

$$
\Im[f \mid g]=4 \int_{\Re^{n}}\left|\left(\nabla+\frac{x}{2}\right) \sqrt{f(x)}\right|^{2} d^{n} x
$$

where $\left(\nabla+\frac{x}{2}\right)$ is the lowering operator of the harmonic oscillator Hamiltonian with ground state $\sqrt{g(x)}$. Here, $g(x)=(2 \pi)^{-\frac{n}{2}} \exp \left[-\frac{x^{2}}{2}\right]$.

Recently, the RFI has been the subject of intense investigations in a quest to obtain a better perspective of its physical implications [10-14], and to formally establish its role in information theory and estimation theory [15, 16]. Still, many of the fundamental properties and physical implications of the RFI remain uninvestigated. The RFI has recently been related to the KullbackLeibler divergence (K-Ld, hereafter)[2]

$$
D[f \| g]=\int_{\Re^{n}} f(\mathbf{x}) \ln \frac{f(\mathbf{x})}{g(\mathbf{x})} d \mathbf{x}
$$


with the aid of the de Bruijn identity [2] by Verdú [15], and Guo, Shamai (Shitz), and Verdú [16] as

$$
\frac{d}{d \delta} D[X+\sqrt{\delta} Z \| Y+\sqrt{\delta} Z]_{\delta=0}=-\frac{1}{2} \Im[X \mid Y]
$$

where $X$ and $Y$ are random variables, and $Z$ is random variable (not necessarily Gaussian), which is independent of $X$ and $Y$. When random variables $X$ and $Y$ have the densities $f$ and $g$, respectively, the K-Ld and the RFI of $X$ with respect to $Y$ are defined by $D[X \| Y]=D[f \| g]$ and $\Im[X \mid Y]=\Im[f \mid g]$, respectively.

Akin to the K-Ld, the RFI may not only be construed as being a measure of uncertainty, but also a measure of discrepancy between two probability densities. The RFI relates to the FIM in a similar manner to which the K-Ld relates to the Shannon entropy. In contrast to the FIM and the RFI, whose derivative term induces the effect of "localization", the Shannon entropy and the K-Ld are "coarse-grained".

It is important to state that this Letter treats the case of one-dimensional time independent probability density functions and their concomitant probability amplitudes. Thus, within the framework of optimal transportation theory [5], the analysis presented herein is applicable to steady-state models. On the other hand, the results of this paper are directly applicable to the RFI models studied in [13-16]. Further, the reference probability $g(\mathbf{x})$ in the expression of the RFI (Eq.(2)) may be treated as representing prior knowledge. Thus, like the K-Ld the RFI possesses the ability of being employed in inference studies.

This Letter accomplishes the following objectives: $(i)$ Setting $g(x)=\exp [-V(x)]$ where $V(x)$ is a convex potential described in Section 2, a principled relation between the RFI, the FIM, and derivative terms of the convex potential $V(x)$ is established (Section 3). (ii) A time independent Schrödinger-like SturmLiouville equation (hereafter referred to as the Schrödinger-like link for the $\mathrm{RFI}$ ), resulting from the variational extremization of the RFI is derived (Section 4). (iii) The reciprocity relations and the Legendre transform structure for the RFI are derived, thereby explicitly demonstrating that the entire mathematical structure of thermodynamics translates into the RFI framework (Sections $5 \& 6$ ). (iv) The utility of the relationship between the RFI and FIM frameworks (derived in Section 3) is demonstrated by inference of the energy eigenvalues of the Schrödinger-like link for the RFI (Section 7). This is achieved by solely utilizing the quantum mechanical virial theorem [17] and the Legendre transform structure of the RFI, without recourse to solving the Schrödinger-like link for the RFI. To the best of the authors' knowledge, none of the above stated results have been hitherto accomplished. 


\section{Theoretical preliminaries}

It is common in optimal transportation theory (eg., see $[5,7,8]$ ) to define the probability $g(\mathbf{x})$ as a reference probability, alternately referred to as the equilibrium probability. Specializing Refs. $[5,7,8]$ and numerous other works to the one-dimensional case

$$
\begin{aligned}
& g(x)=e^{-V(x)}, \\
& \int e^{-V(x)} d x=1,
\end{aligned}
$$

which is known as the Gibbs form, where $V(x)$ is a convex potential.Thus, (2) is re-written with the aid of (6) as

$$
\Im\left[f \mid e^{-V(x)}\right]=\int f(x)|\nabla(\ln f(x)+V(x))|^{2} d x .
$$

It is noteworthy to mention that the term $\ln f(x)+V(x)$ in $(7)$ is exactly the potential $\Psi$ whose expectation in time independent stochastic thermodynamics is an analog to the Helmholtz free energy, for $k_{B} T=$ constant $[18,19]$. Note that in this Letter, all expectation values denoted by $\langle\bullet\rangle$ are evaluated with respect to $f(x)=\psi^{2}(x)$. Here, $\psi(x)$ is the probability amplitude which extremizes the RFI.

\section{Relation between the FIM and the RFI}

Expanding (7) yields

$$
\begin{aligned}
& \Im\left[f \mid e^{-V(x)}\right]=\int\left\{\frac{1}{f(x)}\left(\frac{d f(x)}{d x}\right)^{2}+2 V_{x}(x) \frac{d f(x)}{d x}+V_{x}^{2}(x) f(x)\right\} d x \\
& =\int \frac{1}{f(x)}\left(\frac{d f(x)}{d x}\right)^{2} d x+\int 2 V_{x}(x) \frac{d f(x)}{d x} d x+\int V_{x}^{2}(x) f(x) d x
\end{aligned}
$$

where $V_{x}(x)=\frac{d V(x)}{d x}$. Integrating by parts the second term in the second expression of (8), and specifying $f(x) V_{x}(x)$ to vanish at the boundaries yields

$$
2 \int V_{x}(x) \frac{d f(x)}{d x} d x=-2 \int V_{x x}(x) f(x) d x=-2\left\langle V_{x x}(x)\right\rangle
$$




$$
\begin{aligned}
& V_{x x}(x)=\frac{d^{2} V(x)}{d x^{2}} \text {. Substituting (9) into (8) yields } \\
& \qquad \Im\left[f \mid e^{-V(x)}\right]=\int \frac{1}{f(x)}\left(\frac{d f(x)}{d x}\right)^{2} d x-2\left\langle V_{x x}(x)\right\rangle+\left\langle V_{x}^{2}(x)\right\rangle .
\end{aligned}
$$

Invoking the definition of the FIM in (1) results in the critical relationship

$$
\Im\left[f \mid e^{-V(x)}\right]=I[f]-2\left\langle V_{x x}(x)\right\rangle+\left\langle V_{x}^{2}(x)\right\rangle .
$$

Here, (11) unambiguously relates the RFI with the FIM and the expectations of the derivatives of the convex potential $V(x)$. Thus, (11) tacitly demonstrates that the results presented in this Letter for the RFI qualitatively differ from any results obtained for the FIM (eg. see [3]).

\section{Schrödinger-like link for the RFI}

In physics, it is often desirable to express probabilities in the form of amplitudes. In the one-dimensional case, it is tenable to treat probability amplitudes as real quantities [20]. Expressing (7) in terms of probability amplitudes by specifying $f(x)=\psi^{2}(x)$ and performing variational extremization, results in

$$
\begin{aligned}
& \frac{\delta}{\delta \psi(x)} \int\left\{4\left(\frac{d \psi(x)}{d x}\right)^{2}-2 V_{x x}(x) \psi^{2}(x)+V_{x}^{2}(x) \psi^{2}(x)\right. \\
& \left.-\sum_{i=1}^{M} \lambda_{i} A_{i}(x) \psi^{2}(x)-\lambda_{0} \psi^{2}(x)\right\} d x=0,
\end{aligned}
$$

where $\left\langle A_{i}(x)\right\rangle=\int A_{i}(x) \psi^{2}(x) d x$ are the constraint terms entailing $M$ Lagrange multipliers $\lambda_{i}$, and $\int \psi^{2}(x) d x=1$ is the normalization condition. Carrying through with the variational extremization of (12) yields a time independent Schrödinger-like equation

$$
-\frac{1}{2} \frac{d^{2} \psi(x)}{d x^{2}}-U_{R F I}(x) \psi(x)=\frac{\lambda_{0}}{8} \psi(x) .
$$

The pseudo-potential in (13) comprising of data driven terms, and derivatives of the potential $V(x)$ is defined by

$$
U_{R F I}(x)=\frac{1}{8}\left[\sum_{i=1}^{M} \lambda_{i} A_{i}(x)-V_{x}^{2}(x)+2 V_{x x}(x)\right] .
$$


Note that (13) is a special case of the Sturm-Liouville equation denoted by: $-\frac{d}{d x}\left[q(x) \frac{d \psi(x)}{d x}\right]+g(x) \psi(x)=\mu h(x) \psi(x)$, where $q(x), g(x)$ and $h(x)$ are suitable arbitrary functions, and $\mu$ is the eigenvalue. Specifying: $q(x)=$ $1, g(x)=-U_{R F I}(x), h(x)=1$ and $\mu=\frac{\lambda_{0}}{8}=E$ yields the form of the usual time independent Schrödinger equation, for $\frac{\hbar^{2}}{m}=1$ and having energy eigenvalue $E$.

At this stage it is important to highlight two facts. First, the normalization Lagrange multiplier: $\lambda_{0}=\lambda_{0}\left(\lambda_{1}, \ldots, \lambda_{M}\right)$, and, $\psi(x, \vec{\lambda})$ are the solution of (13), where $\vec{\lambda}$ is a M-vector of Lagrange multipliers. Next, the convex potential $V(x)$ and its derivatives are not functions of $\vec{\lambda}$, and the exact form of $V(x)$ is assumed to be known a-priori. For the sake of generality, the form of $V(x)$ is kept arbitrary in the above analysis. Note that $\psi(x)$ is the probability amplitude that extremizes the RFI. It is interesting to note that setting $g(x)=\exp \left[-\sqrt{k} x^{2}\right]$ results in the potential for the harmonic oscillator, which is one of the most fundamental systems in quantum mechanics.

\section{$5 \quad$ Reciprocity relations}

It is known that standard thermodynamics makes use of derivatives of the Shannon entropy with respect to both parameters, i.e. the Lagrange multipliers $\lambda_{i}$ and expectation values $\left\langle A_{i}(x)\right\rangle$ (for instance, pressure and volume, respectively). The basis for the reciprocity relations is the generalized Euler theorem [21]. In [22], it was shown that the Euler theorem [21] is recovered within the Fisher-context by the formulation of a generalized Fisher-Euler theorem. Given a generic measure of uncertainty $\aleph$ and expectation values $\left\langle A_{i}(x)\right\rangle$, the generalized Euler theorem is required to be of the form [21]

$$
\frac{\partial \aleph}{\partial \lambda_{i}}=\sum_{j=1}^{M} \lambda_{j} \frac{\partial\left\langle A_{j}(x)\right\rangle}{\partial \lambda_{i}}
$$

Here, $\aleph$ may be the Shannon entropy, the FIM, or the RFI (as will be demonstrated in this Section). Specifically, this Section qualitatively extends the analysis in [22] by establishing a principled generalized RFI-Euler theorem. Substituting (14) into (13) and multiplying the resulting expression throughout by 8 yields

$$
-4 \frac{d^{2} \psi(x)}{d x^{2}}-\sum_{i=1}^{M} \lambda_{i} A_{i}(x) \psi(x)-\left[2 V_{x x}(x)-V_{x}^{2}(x)\right] \psi(x)=\lambda_{0} \psi(x)
$$


From [3], the FIM after a single integration by parts is defined by

$$
I[\psi]=4 \int\left(\frac{d \psi(x)}{d x}\right)^{2} d x=-4 \int \psi(x) \frac{d^{2} \psi(x)}{d x^{2}} d x .
$$

Multiplying (16) by $\psi(x)$, integrating and re-arranging the terms results in

$$
-4 \int \psi(x) \frac{d^{2} \psi(x)}{d x^{2}} d x-2\left\langle V_{x x}(x)\right\rangle+\left\langle V_{x}^{2}(x)\right\rangle=\lambda_{0}+\sum_{i=1}^{M} \lambda_{i}\left\langle A_{i}(x)\right\rangle,
$$

where the normalization condition $\int \psi^{2}(x) d x=1$ has been invoked. From (17) and the relation (11) it is readily seen that the LHS of (17) is a re-statement of the RHS of (11) expressed in terms of probability amplitudes, i.e.

$$
\Im\left[\psi \mid e^{-V(x) / 2}\right]=I[\psi]-2\left\langle V_{x x}(x)\right\rangle+\left\langle V_{x}^{2}(x)\right\rangle .
$$

Thus, from (18) and (19) the following relation is obtained

$$
\Im\left[\psi \mid e^{-V(x) / 2}\right]=\lambda_{0}+\sum_{i=1}^{M} \lambda_{i}\left\langle A_{i}(x)\right\rangle,
$$

where $\left\langle A_{i}(x)\right\rangle=\int A_{i}(x) \psi^{2}(x) d x$. Taking the derivative of (20) with respect to $\lambda_{i}$ yields

$$
\frac{\partial \Im\left[\psi \mid e^{-V(x) / 2}\right]}{\partial \lambda_{i}}=\frac{\partial \lambda_{0}}{\partial \lambda_{i}}+\left\langle A_{i}(x)\right\rangle+\sum_{\substack{j=1 \\ j \neq i}}^{M} \lambda_{j} \frac{\partial\left\langle A_{j}(x)\right\rangle}{\partial \lambda_{i}}
$$

Specifying

$$
\frac{\partial \lambda_{0}}{\partial \lambda_{i}}=-\left\langle A_{i}(x)\right\rangle
$$

and substituting (22) into (21) yields

$$
\frac{\partial \Im\left[\psi \mid e^{-V(x) / 2}\right]}{\partial \lambda_{i}}=\sum_{j=1}^{M} \lambda_{j} \frac{\partial\left\langle A_{j}(x)\right\rangle}{\partial \lambda_{i}} .
$$

To demonstrate that the Lagrange multipliers and the expectation values are conjugate variables (see Section 6), it is required to establish: $\frac{\partial \Im\left[\psi \mid e^{-V(x) / 2}\right]}{\partial\left\langle A_{l}(x)\right\rangle}=$ 
$\lambda_{l} ; l=i, j$. While this is evident from (27) in Section 6 , the thermodynamic counterpart of the generalized RFI-Euler theorem (23) is considered by evaluating the derivative of the RFI with respect to the expectation values as

$$
\sum_{i=1}^{M} \frac{\partial \Im\left[\psi \mid e^{-V(x) / 2}\right]}{\partial \lambda_{i}} \frac{\partial \lambda_{i}}{\partial\left\langle A_{j}\right\rangle}=\sum_{i=1}^{M} \sum_{k=1}^{M} \lambda_{k} \frac{\partial\left\langle A_{k}(x)\right\rangle}{\partial \lambda_{i}} \frac{\partial \lambda_{i}}{\partial\left\langle A_{j}(x)\right\rangle}
$$

Eq. (24) readily reduces to

$$
\frac{\partial \Im\left[\psi \mid e^{-V(x) / 2}\right]}{\partial\left\langle A_{j}(x)\right\rangle}=\lambda_{j} \text {, and likewise, } \frac{\partial \Im\left[\psi \mid e^{-V(x) / 2}\right]}{\partial\left\langle A_{i}(x)\right\rangle}=\lambda_{i} \text {. }
$$

The generalized Euler theorem for the Shannon entropy bears similarities to that for the FIM, which has now been shown to bear similarities to that for the RFI. The utility of the generalized Euler theorem is to establish the conjugate relationship between the Lagrange multipliers and the expectation values for a given measure of uncertainty. To establish this conjugate relationship between the Lagrange multipliers and the expectation values for the RFI, it is imperative to utilize Eqs. (23) and (25) above. For the case of the RFI, this conjugate relationship is established in Section 5. In summary, while (23) naturally bears similarities to the form of the generalized Fisher-Euler theorem established in [22] and the Euler theorem within the Boltzmann-Gibbs-Shannon (B-G-S, hereafter) framework [21], it qualitatively differs from the previous studies.

These qualitative distinctions may be summarized as $(i)$ the measure of uncertainty (which in this case is also a measure of discrepancy) is the RFI, (ii) the expectations are evaluated with respect to the probability amplitude $\psi(x)$, which extremizes the RFI, and (iii) the concomitant Lagrange multipliers, which comprise the solution of $(13)$, viz. $\psi(x, \vec{\lambda})$, are dissimilar to those obtained in previous studies. viz. [21, 22]. Specifically, correspondence relationships between the solutions and the Lagrange multipliers of the B-G-S model and the FIM model have been established (eg., see [23])), and the solutions of the variational extremizations of the Shannon entropy and the FIM are known to coincide for the case of equilibrium distributions [3]. Apart from seamlessly relating the RFI and the FIM frameworks, Eq. (11) also provides the basis for relating their respective extremal solutions (and by extension to the extremal solutions of the B-G-S framework) by drawing analogies to the approach adopted in Ref. [23]. This is the objective of ongoing work. 


\section{$6 \quad$ Legendre transform structure}

The objective of obtaining the Legendre transform structure is to place the Lagrange multipliers $\left(\lambda_{i}{ }^{\prime} \mathrm{s}\right)$ and the expectation values $\left(\left\langle A_{i}(x)\right\rangle\right.$ 's) on an equal footing for the purpose of determining $\Im\left[\psi \mid e^{-V(x) / 2}\right]$. This entails that the Lagrange multipliers and the expectation values play reciprocal and symmetric roles thermodynamically, thereby allowing for the input information to be provided also in the form of the Lagrange multipliers. In the usual case, of course, one employs expectation values [24]. Re-stating (20) and re-arranging the terms yields

$$
\lambda_{0}=\Im\left[\psi \mid e^{-V(x) / 2}\right]-\sum_{i=1}^{M} \lambda_{i}\left\langle A_{i}(x)\right\rangle .
$$

From the arguments stated in Section $4, \Im=\Im(x, \vec{\lambda})$. From (26), it is evident that

$$
\lambda_{0}\left(\lambda_{1}, \ldots, \lambda_{M}\right)=\Im\left(\left\langle A_{1}(x)\right\rangle, \ldots,\left\langle A_{M}(x)\right\rangle\right)-\sum_{i=1}^{M} \lambda_{i}\left\langle A_{i}(x)\right\rangle .
$$

Here, (27) is the Legendre transform of the RFI since it changes the identity of the relevant variables; viz. $\Im(x, \vec{\lambda}) \leftrightarrow \Im\left(\left\langle A_{1}(x)\right\rangle, \ldots,\left\langle A_{M}(x)\right\rangle\right)$. Specifically, the Legendre transform relates the input parameters (independent variables of the RFI), such that $\left\{\Im,\left\langle A_{1}(x)\right\rangle, \ldots,\left\langle A_{M}(x)\right\rangle\right\} \leftrightarrow\left\{\lambda_{0}, \vec{\lambda}\right\}$. Note that within the context of (27), $\lambda_{0}$ may also be construed as being the generalized thermodynamic potential of the RFI.

From (27) and (25) (which has been derived on the basis of the generalized RFI-Euler theorem (23)), the following result is obtained

$$
\frac{\partial \lambda_{0}}{\partial \lambda_{i}}=\sum_{j=1}^{M} \frac{\partial \Im}{\partial\left\langle A_{j}(x)\right\rangle} \frac{\partial\left\langle A_{j}(x)\right\rangle}{\partial \lambda_{i}}-\sum_{j=1}^{M} \lambda_{j} \frac{\partial\left\langle A_{j}(x)\right\rangle}{\partial \lambda_{i}}-\left\langle A_{i}(x)\right\rangle=-\left\langle A_{i}(x)\right\rangle .
$$

Eq.'s (25) and (28) yield

$$
\frac{\partial \lambda_{i}}{\partial\left\langle A_{j}(x)\right\rangle}=\frac{\partial \lambda_{j}}{\partial\left\langle A_{i}(x)\right\rangle}=\frac{\partial^{2} \Im}{\partial\left\langle A_{i}(x)\right\rangle \partial\left\langle A_{j}(x)\right\rangle},
$$

and

$$
\frac{\partial\left\langle A_{j}(x)\right\rangle}{\partial \lambda_{i}}=\frac{\partial\left\langle A_{i}(x)\right\rangle}{\partial \lambda_{j}}=-\frac{\partial^{2} \lambda_{0}}{\partial\left\langle A_{i}(x)\right\rangle \partial\left\langle A_{j}(x)\right\rangle},
$$


respectively. As a consequence of (30), the generalized RFI-Euler theorem (23) is re-stated as

$$
\frac{\partial \Im\left[\psi \mid e^{-V(x) / 2}\right]}{\partial \lambda_{i}}=\sum_{j=1}^{M} \lambda_{j} \frac{\partial\left\langle A_{i}(x)\right\rangle}{\partial \lambda_{j}} .
$$

Here, Eqs. (25), (27) and (28)-(31) constitute the Legendre transform structure for the RFI. In essence, (22) and (25) constitute the necessary and sufficient conditions for casting the generalized RFI-Euler theorem (23) in the form specified by (15). Further, (22), (23), and (25) form the basis for establishing the conjugate relationship between the Lagrange multipliers $\left(\lambda_{i}\right.$ 's $)$ and the expectation values $\left(\left\langle A_{i}(x)\right\rangle\right.$ 's). The above results demonstrate the translation of the entire mathematical structure of thermodynamics into the RFI framework. Further, the role of the generalized RFI-Euler theorem (23) in deriving the Legendre transform structure is established.

\section{$7 \quad$ Inference of energy eigenvalues}

The reciprocity relations and Legendre transform structure of the FIM [22] have only employed the data driven information theoretic term $\left\langle A_{i}(x)\right\rangle$ as the expectation value. The Schrödinger-like link for the RFI significantly modifies this scenario by including the derivatives of the convex potential $V(x)$ into the expectation values, since they form an integral part of the potential of the Schrödinger-like link for the RFI. This is evident in Eq's. (13) and (14). It is important to note that while in the FIM model [22], the data driven terms in the pseudo-potential term of the FIM model constitute an informationtheoretic potential, the RFI pseudo-potential (14) also has a physical content owing to the presence of derivatives of $V(x)$ [5]. The leitmotif of this Section is to seamlessly infer the energy eigenvalues of the Schrödinger-like link for the RFI employing only the quantum mechanical virial theorem [19] and the RFI Legendre transform structure (derived in Section 6). This is accomplished without recourse to numerically evaluating the Schrödinger-like link for the RFI. In this context, the work presented in this Section represents a significant qualitative advancement of the analysis in [25].

Multiplying (13) by 2 and re-arranging the terms yields

$$
-\frac{d^{2} \psi(x)}{d x^{2}}+\tilde{U}_{R F I}(x) \psi(x)=\frac{\lambda_{0}}{4} \psi(x)
$$


where the RFI pseudo-potential (14) is re-defined as

$$
\tilde{U}_{R F I}(x)=-\frac{1}{4}\left[\sum_{i=1}^{M} \lambda_{i} A_{i}(x)-V_{x}^{2}(x)+2 V_{x x}(x)\right] .
$$

Note that (32) is of the form of the usual time independent Schrödinger equation having energy eigenvalue $E$, for $\frac{\hbar^{2}}{2 m}=1$ and $\frac{\lambda_{0}}{4}=E$. The quantum mechanical virial theorem for Schrödinger models is [17]

$$
-\int \psi(x) \frac{d^{2} \psi(x)}{d x^{2}} d x=\left\langle x \frac{d \tilde{U}_{R F I}}{d x}\right\rangle
$$

The RFI pseudo-potential (33) is expressed in terms of its physical and data driven components as

$$
\tilde{U}_{R F I}(x)=\tilde{U}_{R F I}^{\text {Physical }}(x)+\tilde{U}_{R F I}^{\text {Data }}(x),
$$

where

$$
\begin{aligned}
& \tilde{U}_{R F I}^{\text {Physical }}(x)=-\frac{1}{4}\left[V_{x}^{2}(x)-2 V_{x x}(x)\right], \\
& \text { and } \\
& \tilde{U}_{R F I}^{\text {Data }}(x)=-\frac{1}{4} \sum_{i=1}^{M} \lambda_{i} A_{i}(x) .
\end{aligned}
$$

Multiplying (34) by 4 and invoking (17) and (35) yields

$$
I[\psi]=4\left\langle x \frac{d \tilde{U}_{R F I}(x)}{d x}\right\rangle=4\left\langle x \frac{d \tilde{U}_{R F I}^{\text {Physical }}(x)}{d x}\right\rangle+4\left\langle x \frac{d \tilde{U}_{R F I}^{\text {Data }}(x)}{d x}\right\rangle
$$

Eq. (37) yields

$$
I[\psi]-4\left\langle x \frac{d \tilde{U}_{R F I}^{\text {Physical }}(x)}{d x}\right\rangle=4\left\langle x \frac{d \tilde{U}_{R F I}^{\text {Data }}(x)}{d x}\right\rangle
$$

Substituting (36) into (38) and invoking (19) yields

$$
\Im\left[\psi \mid e^{-V(x) / 2}\right]=-\sum_{i=1}^{M} \lambda_{i}\left\langle x \frac{d A_{i}(x)}{d x}\right\rangle
$$


Here, (39) requires that the physical pseudo-potential of the Schrödinger-like link for the RFI relates to the convex potential $V(x)$ as

$$
4\left\langle x \frac{d \tilde{U}_{R F I}^{\text {Physical }}(x)}{d x}\right\rangle=2\left\langle V_{x x}(x)\right\rangle-\left\langle V_{x}^{2}(x)\right\rangle
$$

Comparison of (20) and (39) results in

$$
\lambda_{0}+\sum_{i=1}^{M} \lambda_{i}\left\langle A_{i}(x)\right\rangle=-\sum_{i=1}^{M} \lambda_{i}\left\langle x \frac{d A_{i}(x)}{d x}\right\rangle .
$$

The data driven terms in the RFI pseudo-potential may be expressed as moments of the independent variable, because the powers $x^{k}$ constitute a basis in Hilbert space. Thus, without loss of generality one can write

$$
\left\langle A_{i}(x)\right\rangle=\left\langle x^{k}\right\rangle
$$

Invoking now the Legendre transform derived in (28) yields

$$
\frac{\partial \lambda_{0}}{\partial \lambda_{i}}=-\left\langle x^{k}\right\rangle
$$

Substituting (43) into (41) yields the linear PDE

$$
\lambda_{0}=\sum_{i=1}^{M}(1+k) \lambda_{i} \frac{\partial \lambda_{0}}{\partial \lambda_{i}}
$$

The procedure for the inference of the energy eigenvalues of the Schrödingerlike link for the RFI without recourse to the SWE (13) is tacitly encapsulated in the linear PDE (44). Solution of (44) and specifying $\lambda_{0}=4 E$ yields the energy eigenvalues of the the Schrödinger-like link for the RFI. This has only been rendered possible by the invoking of Eq. (19), which is the relationship between the RFI and the FIM. This explicitly demonstrates the immense utility of (19). Apart from constituting a significant condition in its own right, (44) lays the basis for establishing a comprehensive quantal connection for the RFI. Further, (44) requires that the RFI Legendre transform structure be specified in the form derived in Section 6. By induction, this justifies the physical utility of the generalized RFI-Euler theorem (23). 


\section{Summary and discussions}

A critical relation between the RFI and the FIM has been derived (Eq. (11) in Section 3). It has been shown that the mathematical structures underlying thermodynamics can be variationally reproduced by recourse to the RFI. This is accomplished in Section 5, in which a principled generalized RFI-Euler theorem was derived, and in Section 6, in which thermodynamics' Legendre transform structure were expressed in terms of the RFI. The qualitatively distinct nature of these relations within the context of the RFI framework, vis-á-vis prior studies utilizing the B-G-S model and FIM model, have been highlighted. Further, the Schrödinger-like link for the RFI which forms the basis for deriving correspondence relations between the extremal solutions of the RFI and the FIM and/or the Shannon entropy constrained by Lagrange multipliers has been established in Section 5 .

A thermodynamic basis for employing the RFI as a measure of uncertainty in areas such as statistical inference and allied disciplines, parallelling the minimum relative cross entropy principle [26], has been established. Furtherance of this objective comprises the focus of ongoing work. The efficacy of the relation described by (19), which is the manifestation of (11) in the form of probability amplitudes has been highlighted in Section 7. Herein, (19) (and thus (11)) facilitates a principled procedure to infer the energy eigenvalues of the Schrödinger-like link for the RFI (13), with recourse only to the quantum mechanical virial theorem and the Legendre transform structure of the RFI.

Finally, the results of Sections 5 and 7 give rise to an interesting conjecture concerning the nature of the wave function $\psi(x)$, for a physical potential $V(x)$, and, the probability densities $f(x)=\psi^{2}(x)$ and $g(x)=\exp [-V(x)]$ (note that, in one dimension, the wave function can always be taken to be real $[20])$. Here, $\psi(x)$ is the amplitude that extremizes the RFI. Thus, prima facie the RFI associates the pair $\{\psi, V(x)\}$ in a definite manner that does not, in principle, explicitly involve the Schrödinger wave equation. Specifically, given $V(x)$ and performing a search within Hilbert space for the function $\psi(x)$ that extremizes the RFI, it is mathematically feasible to envisage a method of finding $\psi(x)$, without recourse to the Schrödinger-like link for the RFI.

\section{Acknowledgements}

RCV gratefully acknowledges communications with G. Blower. This work was initially conceived under the auspices of $R A N D-M S R$ contract $C S M-D I$ \& $S$-QIT-101155-03-2009, and performed under the auspices of NSFC contract 111017-01-2013. 


\section{References}

[1] R. A. Fisher, Phil. Trans. R. Soc., A, 222 (1922) 309-368.

[2] T. Cover and J. Thomas, Elements of Information Theory, John Wiley \& Sons, New York, NY, 1991.

[3] B. R. Frieden, Science from Fisher Information - A Unification, Cambridge University Press Cambridge, 2004.

[4] B. R. Frieden and R. A. Gatenby(Eds.), Exploratory Data Analysis using Fisher Information, Springer- Verlag, London, 2006.

[5] C. Villani, Topics in Optimal Transportation, Vol.58 Graduate Studies in Mathematics, American Mathematical Society, Providence, R.I., 2003.

[6] G. Blower, Random Matrices: High Dimensional Phenomena, London Mathematical Society Lecture Notes, Cambridge University Press, Cambridge, 2009 .

[7] F. Otto and C. Villani, Journal of Functional Analysis, 173 (2000) 361-400.

[8] U. Gianazza, G. Savaré, G. Toscani, Arch. Ration. Mech. Anal., 194 (2009) $133-220$.

[9] E. Carlen and A. Soffer, Comm. Math. Phys., 140 (1991) 339-371.

[10] T. Yamano, J. Math. Phys., J. Math. Phys., 54 (2013) 113301.

[11] T. Yamano, Eur. J. Phys. B, 86 (2013) 363.

[12] M. Hirata, A. Nemoto, and H. Yoshida, Entropy, 14 (2012) 1469-1477.

[13] P. Sánchez-Moreno, A. Zarzo, and J.S. Dehesa, J. Phys. A: Math. Theor. 45 (2012) 125305.

[14] R. J. Yáñez, P. Sánchez-Moreno, A. Zarzo, and J. S. Dehesa, J. Math. Phys., 49 (2008) 082104.

[15] S. Verdú, IEEE Trans. Inform. Theor., 56 (2010) 3712-3719.

[16] D. Guo, S. Shamai (Shitz) and S. Verdú, The Interplay Between Information and Estimation Measures, Foundations and Trends in Signal Processing Ser., Now Publishers, Boston, 2012.

[17] W. Greiner and B. Müller, Quantum mechanics. An Introduction, Springer, Berlin, 1988.

[18] P. Garbaczewski, Phys. Lett A, 341 (2005) 33-38.

[19] P. Garbaczewski, Acta Phys Pol B, 39 (2008)1087-1101.

[20] A. Messiah, Quantum Mechanics, Dover, New York, NY, 1999. 
[21] A. Katz, Principles of Statistical Mechanics: the Information Theory Approach, W H Freeman, San Francisco, 1967.

[22] B. R.Frieden, A. Plastino, A. R. Plastino, and B. H. Soffer, Phys. Rev. E, 60 (1999) 046128.

[23] M. Casas, F. Pennini, and A. Plastino, Phys. Lett. A, 235 (1997) 457-463.

[24] E. Duering, D. Otero, A. Plastino, and A. N. Proto, Phys. Rev. A, 32 (1985) 2455; Phys. Rev. A, 35 (1987) 2314.

[25] S. P. Flego, A. Plastino, and A. R. Plastino, J. Math. Phys., 52 (2011) 082103.

[26] S. Kullback, Information Theory and Statistics, Wiley, New York, 1959; S. Kullback and M. A. Khairat, Ann. Math. Statist., 37 (1966) 279-280. 